\title{
SAD: Análise da percepção de usuários e desenvolvedores através de análise fatorial
}

\author{
GiLKa Rocha Barbosa \\ Adiel Teixeira de Almeida \\ Ana Paula Cabral Seixas Costa \\ UFPE
}

\begin{abstract}
Resumo
A busca por metodologias que apóiem o desenvolvimento de software tem sido uma preocupação constante. Identificar percepções dos envolvidos é relevante para a construção de software de qualidade. Nesse sentido, este trabalho apresenta um estudo voltado para a identificação da percepção que profissionais desenvolvedores e usuários de sistemas de informação apresentam acerca dos Sistemas de Apoio a Decisão. O levantamento dos dados foi executado contemplando associados à Sociedade de Usuários de Informática e de Telecomunicações (SUCESU) e à Sociedade para Promoção da Excelência do Software Brasileiro [SOFTEX) em Pernambuco. Na avaliação, foram aplicadas técnicas de análise descritiva e análise fatorial, considerando o contexto da atividade profissional dos participantes da pesquisa, o que permitiu identificar características de SAD consideradas relevantes para cada grupo avaliado. O resultado desta avaliação apresenta importantes fatores a serem considerados em metodologias voltadas para o desenvolvimento de SAD.
\end{abstract}

\section{Palavras-chave}

Sistemas de apoio à decisão, Análise fatorial, Análise descritiva, Usuário, Desenvolvedor.

\section{DSS: the perception of users and developers through multivariate statistical techniques}

\begin{abstract}
Adequate methodologies for supporting the development of software have been the object of much research. Identifying the perceptions of the people involved, both developers and users, is an important aspect of quality software construction. This paper presents a study aimed at identifying how information system developers and users perceive Decision Support Systems. The survey was carried out with subjects from the Society of Computer Science and Telecommunications Users (SUCESU) and the Society for the Promotion of Excellence in Brazilian Software [SOFTEX], in the state of Pernambuco, Brazil. The study contains both a descriptive and a factual data analysis with respect to the professional activity of the subject, in order to distinguish the most important characteristics of SAD in each group under study. The result of this evaluation is that the chief factors to be considered in methodologies directed at the development of SAD have been identified.
\end{abstract}

Key words

Decision support systems, Factorial analysis, Descriptive analysis, Decision maker, Information Technology developer. 


\section{INTRODUÇÃO}

A busca por metodologias que apóiem o desenvolvimento dos sistemas de informação tem sido uma constante. Metodologias devem envolver um processo coerente, ordenado e controlável que produza sistemas de qualidade, de forma rápida, com boa documentação, e de fácil manutenção. Essas metodologias vêm evoluindo ao longo do tempo, desde as convencionais, em que a interação entre o analista e o usuário era feita através de uma entrevista, até as atuais, que buscam considerar o usuário como participante de todo o processo de construção do software.

A interação entre analistas e usuários tem sido umas das maiores dificuldades no desenvolvimento de software. Identificar as necessidades do usuário e traduzi-las em linguagens computacionais tem exigido experiência, empenho e sensibilidade. Esse problema se acentua quando se trata de desenvolvimento de sistemas de apoio a decisão (SAD), uma vez que, entre outras características:

- no uso dos SAD não são comuns comportamentos padrões entre o usuário e o aplicativo (o usuário pode incluir fatores e analisar cenários variados, por exemplo);

- a repetição de uma decisão é freqüentemente baixa (o número de vezes de uso do aplicativo é limitado, por exemplo);

- um aplicativo específico pode nunca ser concluído, estando em constante evolução (em geral tratam de decisões não-estruturadas sobre as quais vai-se adquirindo conhecimento aos poucos);

- contam com a necessidade de dados não formais (intuição, experiências e heurística em adição à lógica).

Assim, um SAD deve contar com a participação do usuário em todas as fases do seu ciclo de vida.

Para Pozzebon e Freitas (1997), é necessário que ocorra uma mudança no relacionamento entre os profissionais de sistemas de informação e os usuários para que se alcance a proatividade nos sistemas de informação. Esse é um fator importante para a melhoria da qualidade de software.

Conhecer como os envolvidos com SI vêem e usam os SAD é relevante para que se possa contribuir para um melhor relacionamento entre eles, e apresenta-se como passo inicial para elaboração de propostas de metodologias de desenvolvimento de SI e para maior efetividade na sua utilização, tarefas dificultadas pela natureza complexa dos SAD.

Nesse contexto, foi desenvolvida uma pesquisa com profissionais e usuários de TI acerca de $\mathrm{SAD}$, com vistas a identificar a percepção que esses grupos têm dessa categoria de sistemas, compreendendo e organizando características relevantes para cada um dos grupos. Dessa forma, pode-se contribuir com aspectos relevantes a serem considerados, principalmente, nas etapas de levantamento de requisitos e projeto de interfaces.

Para isso, foi desenvolvido um estudo exploratório através de análise descritiva e análise fatorial - conjunto de técnicas estatísticas para análise multivariada, útil para descobrir regularidades no comportamento entre variáveis estatísticas e para testar modelos alternativos entre elas, incluindo a determinação de quando e como grupos diferem em seu perfil (ARTES, 1998). Assim, aplicadas a dados sobre uso real de sistemas, podem propiciar a identificação, entre outras, das expectativas, práticas de interação e interface, de acordo com os grupos estudados.

\section{Contextualização}

Finlay e Forghani (1998), como resultado de uma pesquisa de campo envolvendo a implantação de 39 SAD, identificaram fatores de sucesso na implantação de um SAD, sendo ressaltados entre os principais: o alinhamento entre o DSS e os requisitos do negócio; e a integração entre usuários e desenvolvedores.

\section{dentificar percepções dos envolvidos na construção e uso é relevante para a construção de software de qualidade}

Em um outro estudo exploratório, Santhanam et al. (2000) concluem que para que um SAD seja realmente eficaz, a observação de alguns aspectos durante as fases de especificação e desenvolvimento é fundamental, entre esses os autores citam: as características do SAD devem ser prioritariamente definidas pelo perfil do usuário, muito mais que pelo problema ou pela tecnologia; a participação efetiva do usuário no desenvolvimento e implementação do SAD é fundamental, além de um completo treinamento na ferramenta; e outro aspecto relevante é o envolvimento do desenvolvedor em todo o processo que antecede a especificação do SAD.

Ainda ressaltando a importância do usuário no processo de implantação de um SAD, Chen e Lee (2003) enfatizam que uma das razões que podem comprometer a eficácia de um SAD é a negligência com os aspectos cognitivos do $\mathrm{SAD}$, argumentando que, embora vários $\mathrm{SAD}$ forneçam aos seus usuários modelagens quantitativas e rapidez de acesso a dados, os aspectos cognitivos não têm sido levados em consideração em seus projetos.

Observa-se, pelo encontrado na literatura, que as divergências de percepção entre usuários e desenvolvedores podem comprometer o desempenho de um SAD, contudo, é consenso que a utilização de SAD apóia processos de tomada de decisão, fornecendo ao tomador de decisão mais detalhes acerca 
do problema e melhorando seu entendimento sobre as conseqüências das possíveis decisões e mudanças no ambiente (FUGLSETH; GRONHAUG, 2003), contudo, como é possível avaliar o quão eficaz é um sistema de apoio à decisão?

Fazlollahi et al. (1997) dizem que o tomador de decisão é determinante na avaliação da eficácia dos SAD, e que esta está entrelaçada ao processo adaptativo de apoio às suas necessidades. Os autores afirmam que o desenho e a sistema seja eficaz. Desde o início do processo de criação, o sistema precisa ser adaptado às necessidades para atender às mudanças do ambiente. A alta adaptabilidade adiciona funcionalidade ao SAD. implantação de um SAD bem-feitos não garantem que o

\section{Descrição da pesquisa}

O estudo apresentado neste trabalho teve como principal objetivo identificar a percepção que os desenvolvedores e usuários apresentam acerca de SAD.

O primeiro passo foi à criação de uma estrutura de trabalho com as características mais gerais encontradas na literatura, voltadas para categorizar SAD quanto ao seu uso, interface, tecnologia e pessoal envolvido, com vistas a auxiliar na elaboração da pesquisa. A estrutura é, portanto, produto de uma cuidadosa análise e síntese de várias fontes, tais como: Alter (1994), Bidgoli (1989), Courtney (2001), Davis (1974), Garnto (1991), Laudon e Laudon (1999), Sprague e Watson (1989), e Stair (1998).

A estrutura, além de sistematizar conceitos referentes aos SAD, estabeleceu referenciais para elaboração do instrumento de pesquisa. Resumidamente, o Quadro 1 mostra variáveis e características desejáveis para um $\mathrm{SAD}$, conforme a literatura.

Os dados foram coletados a partir de questionários respondidos por

Um dos principais problemas em determinar fatores do sucesso do SAD é a dificuldade em estabelecer medidas apropriadas de sucesso (FINLAY; FORGHANI, 1998). O sistema de controle de qualidade de um SAD é ainda muito subjetivo, estabelecer medidas padronizadas para verificar se o sistema atende às especificações não é muito usual. Como medidas, podem ser citadas: usabilidade (se o sistema é ou foi realmente utilizado), maior rapidez na tomada de decisão, melhor comunicação, melhor entendimento do problema, entre outras. A questão é que essas medidas variam de acordo com o tipo de sistema e, portanto, são difíceis de ser determinadas.

Um dos motivos que reforçam a necessidade do SAD ser um sistema adaptável é o fato de que é difícil predizer todos os possíveis cenários que o usuário terá, tendo-se certeza de que haverá mudanças e evolução no ambiente onde o problema está inserido.

Pode-se perceber, pelo exposto, que o desenvolvimento de um SAD é uma tarefa difícil, uma vez que não se consegue definir suas necessidades funcionais antecipadamente. Outro fator importante é que o tomador de decisão e o desenvolvedor adquirem conhecimento acerca do problema ao longo do processo de desenvolvimento do SAD (ALVIM, 2002).

Não é difícil perceber que uma metodologia de desenvolvimento de SI deve estimular a relação entre o desenvolvedor e o usuário. Esses aspectos, assim como a natureza do SAD, voltam-se mais uma vez para a necessidade de entender o estilo cognitivo do usuário, que tem sido um dos fatores considerados relevantes para compreensão das diferenças individuais no uso de SAD. profissionais associados à SUCESU, entidade que congrega empresas de destaque no desenvolvimento e utilização de SI, e a SOFTEX, organização voltada para a excelência na produção de software. Os questionários foram enviados aos profissionais que exerciam funções de gerência ou que trabalhavam no desenvolvimento de software. Os grupos foram avaliados separadamente, em seguida as informações obtidas foram cruzadas e comparadas.

As variáveis foram organizadas em aspectos necessários para conhecer práticas referentes ao desenvolvimento e à utilização de SAD. A Figura 1 apresenta estes aspectos utilizados para identificar a percepção de SAD. Os profissionais que exerciam funções de gerência e os desenvolvedores de software foram questionados sobre como são desenvolvidos e utilizados os $\mathrm{SAD}$, qual tecnologia é utilizada nesse desenvolvimento e quais as melhores práticas de desenvolvimento e uso. Além disso, foram realizadas questões sobre o processo decisório, envolvendo aspectos da tomada de decisão e a relação entre a decisão e o SAD.

A relativa juventude do tema conduziu a uma pesquisa exploratória, mais apropriada para estágios iniciais de investigação (MATTAR, 1996) e indicada para descobertas de idéias e intuição, aumentar o conhecimento da situação, esclarecer conceitos ou obter informações sobre possibilidades práticas de realização de pesquisas em situações de vida real. O método utilizado na pesquisa foi o estudo da experiência, que considera um levantamento de pessoas que tiveram experiência prática como o problema a ser estudado, pois adquirem na rotina do trabalho um extraordinário conjunto de experiências que pode ter muito valor para que sejam 
Quadro 1: Variáveis desejáveis para um SAD.

\begin{tabular}{|c|c|}
\hline VARIÁVEL & CARACTERÍSTICA DESEJÁVEL \\
\hline Origem dos dados & Dados podem ser originados nos sistemas internos ou no ambiente externo à empresa. \\
\hline Base de dados & $\begin{array}{l}\text { Deve ser separada do banco de dados do processamento de transações e lidar com dados } \\
\text { não-oficiais, pessoais, julgamentos etc. }\end{array}$ \\
\hline Tipos de dados & Capacidade de combinar, acrescentar ou excluir fontes de dados. \\
\hline $\begin{array}{l}\text { Estruturação do } \\
\text { problema }\end{array}$ & Auxílio nas decisões com problemas de estruturação. \\
\hline Níveis hierárquicos & Em todos os níveis de gerenciamento da empresa. \\
\hline $\begin{array}{l}\text { Fases do processo } \\
\text { decisório }\end{array}$ & Identificação do problema, propostas de solução e escolha de alternativa. \\
\hline Tipos de operação & $\begin{array}{l}\text { Acesso a itens simples, mecanismo ad hoc, análise e comparações complexas, combinação } \\
\text { de uso de modelos. }\end{array}$ \\
\hline Seqüência de execução & $\begin{array}{l}\text { Flexíveis, independentes do processo, controlados pelo usuário e direcionados a ele. Inter- } \\
\text { relacionamento e criação de novos modelos. }\end{array}$ \\
\hline $\begin{array}{l}\text { Adaptação a novas } \\
\text { necessidades }\end{array}$ & Criação fácil e rápida de novos modelos. \\
\hline Interface amigável & $\begin{array}{l}\text { Fáceis de usar, capacidade de apresentar dados de diversos formatos e suporte flexível ao } \\
\text { banco de conhecimento do usuário. }\end{array}$ \\
\hline Operação do sistema & $\begin{array}{l}\text { Recursos que facilitam seu uso por pessoal não especializado em computação de forma } \\
\text { iterativa. }\end{array}$ \\
\hline Freqüência de uso & $\begin{array}{l}\text { Podem ser institucionais, voltados para problemas que não se antecipam ou não } \\
\text { recorrentes, que apresentam modelos e séries de dados incompatíveis e diversos, ad hoc. }\end{array}$ \\
\hline Autonomia do usuário & Usados de uma forma ativa, possibilitam desenvolvimento e ajustes por parte do usuário. \\
\hline $\begin{array}{l}\text { Fatores que influenciam } \\
\text { na decisão }\end{array}$ & Servem apara apoiar o tomador de decisão e não para substituí-lo. \\
\hline $\begin{array}{l}\text { Desenvolvimento do } \\
\text { sistema }\end{array}$ & O usuário deve participar de todas as fases do desenvolvimento do sistema. \\
\hline
\end{tabular}

Figura 1: Aspectos utilizados para identificar a percepção de SAD.

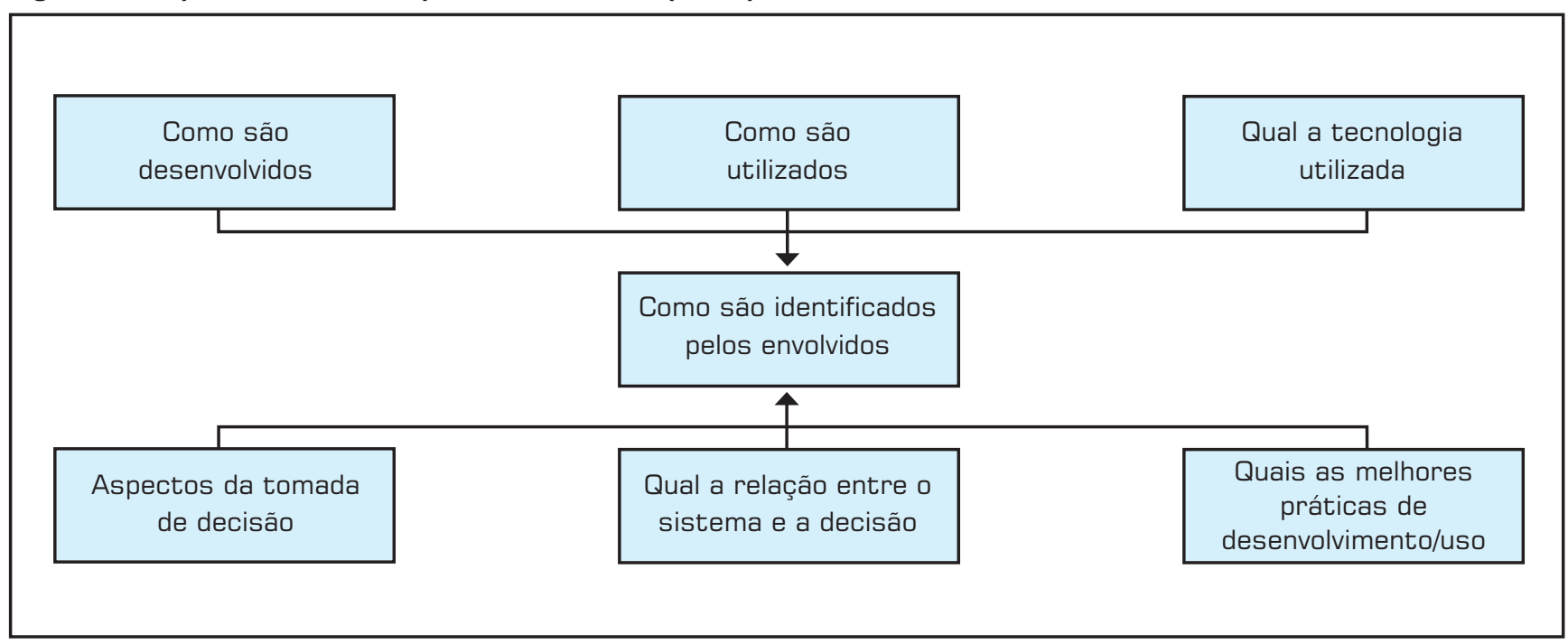


conhecidas importantes influências que atuam na situação estudada (SELLTZ, 1967).

Os dados do experimento foram analisados por meio de técnicas estatísticas (análise descritiva e análise fatorial), por meio do software SPSS for Windows, versão 10.0 - 1999.

A análise descritiva dos dados voltou-se para a descrição do comportamento de cada uma das categorias profissionais com as variáveis da estrutura referencial proposta.

Através da análise fatorial buscou-se identificar a percepção de SAD pelos envolvidos. Esta técnica considera que, a partir de uma estrutura de dependência existente entre variáveis (variáveis originais), é possível a criação de um conjunto menor de variáveis (variáveis latentes, resultantes ou fatores), e saber que fatores associam as variáveis resultantes a cada variável original. O método utilizado para obtenção dos fatores foi o de componentes de fatores. A carga fatorial expressa o quanto cada variável original está associada a cada fator, e os autovalores refletem a importância de cada um dos fatores envolvidos, podendo revelar agrupamentos ou tendências que poderiam ser difíceis de visualizar nas variáveis originais. $\mathrm{O}$ critério utilizado na determinação do número de fatores foi o Critério de Kaiser, que determina que o número de fatores deve ser igual ao de autovalores maiores ou iguais a um. A análise fatorial foi exploratória, que não exige formulação de hipótese a priori acerca da estrutura dos dados. Essa estrutura, se existir, será um dos seus resultados (ARTES, 1998).

Cada técnica utilizada é um conjunto de tarefas e ambientes que medem o resultado da combinação de técnicas componentes e apresentam fatores para múltiplas variáveis. As técnicas podem testar, bem como criar, taxonomias e hipóteses a partir dos dados.

\section{Análise descritiva exploratória}

Com o objetivo de conhecer como os SAD são utilizados nas empresas, foram levantados dados acerca das organizações, tais como atividade, setor, porte, área e mercado de atuação da empresa. Sobre os respondentes, obtiveram-se dados sobre o grau de escolaridade e formação acadêmica. Esses dados encontram-se detalhados em Barbosa (2003).

Dos 66 questionários recebidos, 9 responderam que não conheciam ou não usavam $\mathrm{SAD}$ e não foram considerados na pesquisa. Os respondentes considerados nesta análise são apresentados na Tabela 1.

Uma das principais características dos SAD refere-se à estruturação do problema a ser tratado. Conforme ressaltado por Almeida et al. (2002), o nível de estruturação do problema cuja solução é apoiada pelos sistemas de informação é fundamental para identificar a abordagem adequada a esses sistemas, principalmente, para diferenciar sistemas de informação gerencial (SIG) e SAD, uma vez que ambos se prestam a apoiar na tomada de decisões. Nesse contexto, foram considerados para análise apenas os sistemas informados pelos respondentes que apresentassem o nível de estruturação do problema como semi-estruturado ou nãoestruturado. A Tabela 2 mostra o nível de estruturação dos sistemas considerados na pesquisa.

Tabela 1: Respondentes por atividade.

\begin{tabular}{|l|c|c|}
\hline \multicolumn{1}{|c|}{ PROFISSIONAL } & NÚMERO & FREQÜÊNCIA \\
\hline Desenvolvedores & 35 & $61,4 \%$ \\
\hline Usuários & 22 & $38,6 \%$ \\
\hline Total observado & 57 & $100,0 \%$ \\
\hline
\end{tabular}

Tabela 2: Nível de estruturação do problema.

\begin{tabular}{|l|c|c|c|c|}
\hline \multirow{2}{*}{ NíVEL DE ESTRUTURAÇÃO DO PROBLEMA } & \multicolumn{2}{|c|}{ DESENVOLVORES } & \multicolumn{2}{|c|}{ USUÁRIOS } \\
\cline { 2 - 5 } & NÚMERO & FREQÜÊNCIA & NÚMERO & FREQÜÊNCIA \\
\hline Semi-estruturado & 20 & $74,1 \%$ & 15 & $100,0 \%$ \\
\hline Não-estruturado & 7 & $25,9 \%$ & 0 & $0,0 \%$ \\
\hline Total observado & 27 & $100,0 \%$ & 15 & $100,0 \%$ \\
\hline
\end{tabular}

Tabela 3: Origem dos dados.

\begin{tabular}{|l|c|c|c|c|}
\hline \multirow{2}{*}{ ORIGEM DOS DADOS } & \multicolumn{2}{|c|}{ DESENVOLEDORES } & \multicolumn{2}{c|}{ USUÁRIOS } \\
\cline { 2 - 5 } & NÚMERO & FREQÜÊNCIA & NÚMERO & FREQÜÊNCIA \\
\hline Interna à empresa & 10 & $37,0 \%$ & 5 & $33,3 \%$ \\
\hline Externa à empresa & 1 & $3,7 \%$ & 1 & $6,7 \%$ \\
\hline Ambos & 16 & $59,3 \%$ & 9 & $60,0 \%$ \\
\hline Total observado & 27 & $100,0 \%$ & 15 & $100,0 \%$ \\
\hline
\end{tabular}


Analisando a Tabela 3, pode-se dizer que, quanto à origem de dados, a maioria dos sistemas apresenta característica relevante na definição de SAD, qual seja, processamento de dados não apenas de fontes internas à empresa.

Quanto à base de dados (Tabela 4), observa-se uma característica de definição de SAD: a maioria dos sistemas possui sua própria base de dados. É menor a incidência de sistemas que manipulam outros tipos de dados, tais como percepções e estimativas, e merece destaque a quantidade de sistemas que apenas acessam a base de dados transacional da empresa.

Ainda relacionada à questão dos dados processados, a Tabela 5 apresenta como o sistema é alimentado. A possibi- lidade de variar os dados de entrada é um aspecto importante para a flexibilidade de uso do sistema, e uma característica importante nos SAD.

O nível hierárquico organizacional para o qual o sistema é desenvolvido é apresentado na Tabela 6. Esses dados conduzem a uma reflexão sobre a visão dos desenvolvedores quanto à utilização dos sistemas, pois levam como pode um sistema ao apoiar decisão em nível operacional, tático e estratégico ao mesmo tempo? Uma vez que apresentam características da tomada de decisão diferentes em relação a abrangência, tipo, efeito e escala tempo, diferenças essas que para um sistema de informação atender seria extremamente complexo.

Tabela 4: Base de dados.

\begin{tabular}{|l|c|c|c|c|}
\hline \multicolumn{1}{|c|}{ BASE DE DADOS } & \multicolumn{2}{c|}{ DESENVOLEDORES } & \multicolumn{2}{c|}{ USUÁRIOS } \\
\cline { 2 - 5 } & NÚMERO & FREQÜÊNCIA & NÚMERO & FREQÜÊNCIA \\
\hline Base de dados transacional & 4 & $14,8 \%$ & 2 & $13,3 \%$ \\
\hline Possui sua própria base de dados & 9 & $33,3 \%$ & 7 & $46,7 \%$ \\
\hline $\begin{array}{l}\text { Base de dados transacional e } \\
\text { Possui sua própria base de dados }\end{array}$ & 3 & $11,1 \%$ & 3 & $20,0 \%$ \\
\hline $\begin{array}{l}\text { Possui sua própria base de dados e } \\
\text { Permite outros dados, tais como estimativas pessoais }\end{array}$ & 7 & $26,9 \%$ & 1 & $6,7 \%$ \\
\hline $\begin{array}{l}\text { Base de dados transacional, } \\
\text { Possui sua própria base de dados e } \\
\text { Permite outros dados, tais como estimativas pessoais }\end{array}$ & 4 & & & \\
\hline Total observado & 27 & $100,0 \%$ & 15 & $100,0 \%$ \\
\hline
\end{tabular}

Tabela 5: Tipo de dados.

\begin{tabular}{|l|c|c|c|c|}
\hline \multirow{2}{*}{ TIPOS DE DADOS } & \multicolumn{2}{c|}{ DESENVOLVDORES } & \multicolumn{2}{c|}{ USUÁRIOS } \\
\cline { 2 - 5 } & NÚMERO & FREQÜÊNCIA & NÚMERO & FREQÜÊNCIA \\
\hline Predefinidos & 9 & $33,3 \%$ & 6 & $40,0 \%$ \\
\hline Definidos de acordo com a necessidade & 18 & $66,7 \%$ & 9 & $60,0 \%$ \\
\hline Total observado & 27 & $100,0 \%$ & 15 & $100,0 \%$ \\
\hline
\end{tabular}

Tabela 6: Níveis hierárquicos que utilizam o sistema.

\begin{tabular}{|l|c|c|c|c|}
\hline \multirow{2}{*}{ NÍVEIS HIERÁRQUICOS QUE USAM O SISTEMA } & \multicolumn{2}{|c|}{ DESENVOLEORES } & \multicolumn{2}{c|}{ USUÁRIOS } \\
\cline { 2 - 5 } & NÚMERO & FREQÜÊNCIA & NÚMERO & FREQÜÊNCIA \\
\hline Operacional & 3 & $11,1 \%$ & 2 & $13,3 \%$ \\
\hline Tático & 3 & $11,1 \%$ & 2 & $13,3 \%$ \\
\hline Operacional e tático & 3 & $11,1 \%$ & 1 & $6,7 \%$ \\
\hline Tático e estratégico & 9 & $33,3 \%$ & 8 & $53,3 \%$ \\
\hline Operacional, tático e estratégico & 9 & $33,3 \%$ & 2 & $13,3 \%$ \\
\hline Total observado & 27 & $100,0 \%$ & 15 & $100,0 \%$ \\
\hline
\end{tabular}


A fim de conhecer mais detalhadamente os sistemas considerados como $\mathrm{SAD}$, foram questionadas as fases do processo decisório apoiadas pelo sistema (Tabela 7).

Embora seja um aspecto relevante na sua definição, a natureza flexível e adaptável dos SAD foi pouco ressaltada nos dados. Observando-se a Tabela 8, percebe-se que a maioria dos sistemas classificados como SAD, por ambas as categorias de profissionais pesquisadas, não possui flexibilidade de execução de rotinas e sub-rotinas. Ou seja, não utilizam com freqüência modelos alternativos para a solução de problemas. Esse percentual pode significar que os sistemas atuais mantêm características dos convencionais.

Quanto à forma de operação, a Tabela 9 mostra que a maioria dos sistemas é operada pelo próprio usuário. Quando não, as funções que mais aparecem na intermediação entre o aplicativo e o usuário foram analista de negócios, analista e auxiliar de planejamento.

Quanto aos sistemas, foram avaliados ainda aspectos acerca da freqüência de utilização do sistema, grau de autonomia, e das principais dificuldades encontradas na operação do sistema pelo usuário.

Quanto à tomada de decisão, percebeu-se que, além das informações geradas pelo sistema, a maioria dos usuários utiliza a intuição e a experiência. Além disso, os desenvolvedores consideram o sistema como o fator de maior contribuição para a tomada de decisão, enquanto que os usuários mostram que o sistema de informação e a sua experiência contribuem na mesma proporção para a solução do problema.

No tocante ao desenvolvimento do sistema, constatou-se que é pequena a participação do usuário em sistemas adquiridos prontos (pacotes), embora a customização necessária exija sua participação direta no planejamento e desenvolvimento. Quanto às funções envolvidas no desenvolvimento, os entrevistados asseguram que, na maioria das vezes, usuário e analistas ou programadores participam do desenvolvimento. Boa parte dos sistemas apresentados pelos usuários é desenvolvida por eles próprios, significando autonomia de desenvolvimento das suas soluções.

Acerca do nível de satisfação do usuário, os respondentes afirmaram que os sistemas proporcionam aos usuários um bom nível de satisfação.

Foram ainda questionadas a flexibilidade dos sistemas, a amigabilidade da interface e a tecnologia no uso e desenvolvimento do sistema (BARBOSA, 2003).

A análise descritiva mostra que os desenvolvedores tendem a definir SAD como SI que possam ser manipulados pelo usuário, não priorizam mecanismos voltados para modelos alternativos, vêem como exceção SI voltados para problemas não rotineiros e que não apresentem rotinas

Tabela 7: Fases do processo decisório.

\begin{tabular}{|l|c|c|c|c|}
\hline \multicolumn{1}{|c|}{$\begin{array}{c}\text { FASES DO PROGESSO DECISÓRIO } \\
\text { APOIADAS PELO SISTEMA }\end{array}$} & \multicolumn{1}{c|}{ DESENVOLEDORES } & \multicolumn{2}{c|}{ USUÁRIOS } \\
\cline { 2 - 5 } & NÚMERO & FREQÜÊNCIA & NÚMERO & FREQÜÊNCIA \\
\hline Identificação de problemas & 11 & $40,7 \%$ & 3 & $20,0 \%$ \\
\hline Proposta de soluções alternativas & 1 & $3,7 \%$ & 3 & $20,0 \%$ \\
\hline Seleção de solução & 1 & $3,7 \%$ & 1 & $6,7 \%$ \\
\hline Não sabe & 2 & $7,4 \%$ & 1 & $6,7 \%$ \\
\hline Identificação de problemas e Proposta de soluções alternativas & 0 & $0,0 \%$ & 4 & $26,7 \%$ \\
\hline Identificação de problemas e Seleção de solução & 2 & $7,4 \%$ & 1 & $6,7 \%$ \\
\hline Proposta de soluções alternativas e Seleção de solução & 8 & $29,6 \%$ & 2 & $13,3 \%$ \\
\hline $\begin{array}{l}\text { Identificação de problemas, Proposta de soluçães alternativas } \\
\text { e Seleção de solução }\end{array}$ & 2 & $7,4 \%$ & 0 & $0,0 \%$ \\
\hline Total observado & 27 & $100,0 \%$ & 15 & $100,0 \%$ \\
\hline
\end{tabular}

Tabela 8: Seqüência de operação das rotinas do sistema.

\begin{tabular}{|l|c|c|c|c|}
\hline \multirow{2}{*}{$\begin{array}{c}\text { SEQÜÊNCIA DE EXECUÇÄO } \\
\text { DAS ROTINAS }\end{array}$} & \multicolumn{2}{|c|}{ DESENVOLVEORES } & \multicolumn{2}{c|}{ USUÁRIOS } \\
\cline { 2 - 5 } & NÚMERO & FREQÜẾNCIA & NÚMERO & FREQÜÊNCIA \\
\hline Modelos preestabelecidos & 15 & $55,6 \%$ & 13 & $86,7 \%$ \\
\hline Modelos conforme a necessidade & 12 & $44,4 \%$ & 2 & $13,3 \%$ \\
\hline Total observado & 27 & $100,0 \%$ & 15 & $100,0 \%$ \\
\hline
\end{tabular}


sistemáticas, e, mesmo considerando o sistema como apoio a uma decisão, excluem algumas vezes o usuário do seu desenvolvimento. Esses sistemas apóiam todos os níveis organizacionais, e são orientados a dados, através de análise ad hoc e agregação de dados em procedimentos predefinidos. São voltados para identificação de problemas, adaptáveis a novas necessidades, e apresentam interface amigável.

Os usuários identificam SAD como SI alimentados a partir de fontes internas e externas à organização, que possuam sua própria base de dados, utilizados como apoio aos níveis táticos e estratégicos, voltados para a identificação de problemas e proposta de soluções, adaptáveis a novas necessidades e com interface amigável, que priorizem a manipulação de dados de forma flexível. E, embora alguns desenvolvam suas próprias soluções, em geral, não dispensam o desenvolvedor no desenvolvimento do sistema.

A Quadro 2 apresenta, como resultado da análise descritiva, um resumo com as principais características dos $\mathrm{SAD}$ determinadas pelos grupos respondentes.

Os principais aspectos em comum percebidos entre os sistemas apresentados e a definição de $\mathrm{SAD}$, segundo a literatura, dizem respeito à flexibilidade de manipulação da base de dados e à estruturação do problema; entretanto, não apresentam flexibilidade quanto à execução ativa e ao uso de modelos.

\section{Análise fatorial exploratória}

Com o objetivo de categorizar aspectos de um SAD segundo os grupos envolvidos na pesquisa e devido ao grande número de variáveis originais, os dados foram submetidos ao tratamento estatístico através de uma análise fatorial.

\section{s desenvolvedores priorizam forma de operação, flexibilidade de alimentação e facilidade de atualização do sistema.}

Inicialmente, foram definidas como variáveis originais para análise: Nível de estruturação, Origem dos dados, Base de dados, Tipo de dados, Seqüência de execução das rotinas, Grau de adaptação a novas necessidades, Grau de amigabilidade da interface e Grau de autonomia do usuário. Essas variáveis, apresentadas no Quadro 3, foram escolhidas por apresentarem, individualmente, características desejáveis aos SAD. Como para esta análise foram selecionados apenas os sistemas voltados para problemas não-estruturados ou semiestruturados (os que atribuíram valores Semi-estruturado ou Não-estruturado à variável Nível de estruturação), essa variável na se encontra explicitada junto às outras na análise.

Definidas as variáveis, os dados foram submetidos à análise pelo método de extração de componentes de fatores, conforme a atividade profissional.

Quadro 2: Características dos SAD pesquisados.

\begin{tabular}{|l|l|}
\hline \multicolumn{1}{|c|}{ DESENVOLVEOR } & USUÁRIO \\
\hline Manipulam dados internos e externos & Idem \\
\hline Possuem base própria, mas não permitem outros tipos de dados & Idem \\
\hline Manipulam entradas, mas não incluem ou excluem fontes de dados & Idem \\
\hline Apóiam problemas semi-estruturados & Idem \\
\hline Apóiam a todos os níveis simultaneamente & Tático e o estratégico \\
\hline Apóiam a seleção de soluções & Identificação de problemas e propostas de solução \\
\hline $\begin{array}{l}\text { Acesso a itens simples, questões ad hoc e procedimentos } \\
\text { predefinidos. }\end{array}$ & Idem \\
\hline Não apresentam flexibilidade de modelos & Idem \\
\hline Apresentam alto grau de adaptabilidade & Médio \\
\hline Apresentam uma boa interface & Idem \\
\hline Operados pelos usuários & Idem \\
\hline Usados diariamente & Idem \\
\hline Proporcionam autonomia regular & Boa autonomia. \\
\hline Usados junto à intuição e à experiência & Juntam-se ao uso de intuição, experiência e literatura \\
\hline Desenvolvidos pelos programadores e usuários & Idem \\
\hline
\end{tabular}


Os fatores encontrados para cada tipo de atividade de profissional são discutidos a seguir. Cada um desses fatores é representado por um gráfico formado pelas variáveis originais que mais contribuíram para a sua definição.

\section{Desenvolvedores}

Aplicando o método de extração das componentes de fatores a partir da matriz de correlações, após rotação Varimax, foi gerado o carregamento fatorial conforme Figura 2, onde são apresentados os autovalores e possíveis fatores encontrados.

Figura 2: Autovalores para desenvolvedores.

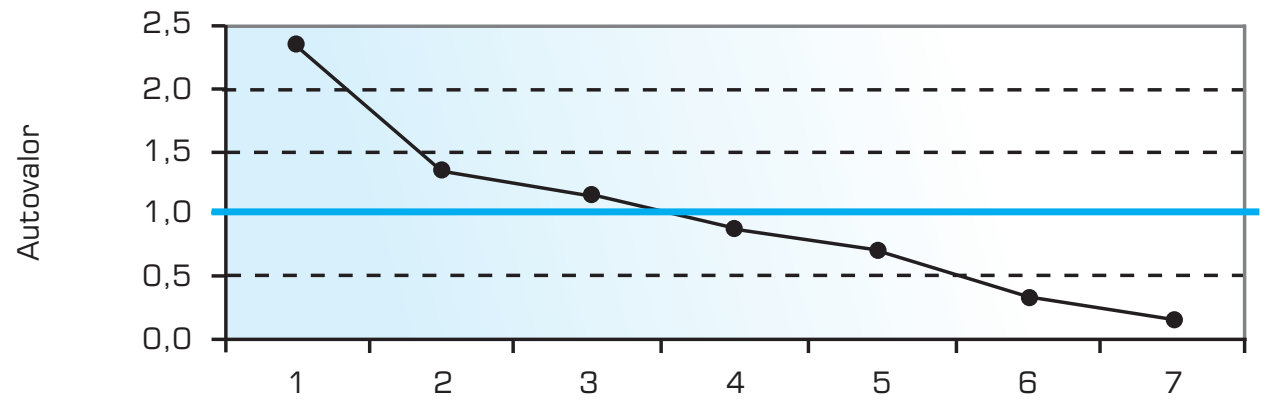

Fator

Quadro 3: Principais variáveis de definição de SAD.

\begin{tabular}{|c|c|c|c|}
\hline \multirow{2}{*}{ VARIÁVEL } & \multicolumn{2}{|r|}{ VALORES ATRIBUÍDOS } & \multirow{2}{*}{\begin{tabular}{|c} 
CARAGTERÍSTICAS \\
PREDOMINANTES NA LITERATURA
\end{tabular}} \\
\hline & NUMÉERICO & IDENTIFIGAÇÃO & \\
\hline Origem de dados & $\begin{array}{l}1 \\
2 \\
3\end{array}$ & $\begin{array}{l}\text { Interna } \\
\text { Externa } \\
\text { Ambos }\end{array}$ & $\begin{array}{l}\text { Externa } \\
\text { Ambos }\end{array}$ \\
\hline Base de dados & $\begin{array}{l}1 \\
2 \\
3\end{array}$ & $\begin{array}{l}\text { Base transacional } \\
\text { Base própria } \\
\text { Outros dados }\end{array}$ & $\begin{array}{l}\text { Base própria } \\
\text { Outros dados (tais como } \\
\text { estimativas pessoais) }\end{array}$ \\
\hline Tipos de dados & $\begin{array}{l}1 \\
2\end{array}$ & $\begin{array}{l}\text { Predefinido } \\
\text { Definido de acordo com a necessidade }\end{array}$ & De acordo com a necessidade \\
\hline $\begin{array}{l}\text { Seqüência de operação } \\
\text { das rotinas }\end{array}$ & $\begin{array}{l}1 \\
2\end{array}$ & $\begin{array}{l}\text { Modelo preestabelecido } \\
\text { Modelo conforme a necessidade }\end{array}$ & Modelo conforme a necessidade \\
\hline Grau de adaptação & $\begin{array}{l}1 \\
2 \\
3\end{array}$ & $\begin{array}{l}\text { Baixo } \\
\text { Médio } \\
\text { Alto }\end{array}$ & $\begin{array}{l}\text { Médio } \\
\text { Alto }\end{array}$ \\
\hline $\begin{array}{l}\text { Amigabilidade da } \\
\text { interface }\end{array}$ & $\begin{array}{l}1 \\
2 \\
3 \\
4 \\
5\end{array}$ & $\begin{array}{l}\text { Péssimo } \\
\text { Ruim } \\
\text { Regular } \\
\text { Bom } \\
\text { Excelente }\end{array}$ & $\begin{array}{l}\text { Regular } \\
\text { Bom } \\
\text { Excelente }\end{array}$ \\
\hline Autonomia de uso & $\begin{array}{l}1 \\
2 \\
3 \\
4 \\
5\end{array}$ & $\begin{array}{l}\text { Péssimo } \\
\text { Ruim } \\
\text { Regular } \\
\text { Bom } \\
\text { Excelente }\end{array}$ & $\begin{array}{l}\text { Regular } \\
\text { Bom } \\
\text { Excelente }\end{array}$ \\
\hline
\end{tabular}


Considerando o critério de Kaizer, foram selecionados os três fatores com autovalor maior que 1 (os pontos que se encontram acima da linha azul da Figura 2), que juntos explicam $71,20 \%$ da variância dos dados, descritos na Tabela 10. Apesar de possível, um $4^{\circ}$ fator não foi considerado, pois o objetivo foi obter o menor número possível de variáveis latentes para a representação.

A Tabela 11 apresenta as cargas fatoriais, a partir das quais foi escolhido para interpretação de cada fator o conjunto de variáveis originais que apresentam o maior peso (destacadas em vermelho).

O Fator 1, que explica 34,32\% da variação dos dados, apresenta como maior carga fatorial as variáveis Base de dados, Tipo de dados e Seqüência de execução das rotinas, que podem ser reconhecidas como habilidade para operação do SI para solução do problema. O Fator 2 explica 19,66\% da variação dos dados e apresenta uma maior contribuição das variáveis Origem dos dados e Grau de amigabilidade do sistema, que podem juntas avaliar aspectos da flexibilidade de alimentação do sistema. O Fator 3 representa 17,21\% da variação dos dados e confirma as variáveis Grau de adaptação a novas necessidades e Nível de autonomia no uso do sistema, que juntas podem indicar a autonomia na atualização do sistema.

Com estes resultados, tem-se o delineamento de três fatores representando características do $\mathrm{SAD}$, que podem ser denominadas Operação, Alimentação e Atualização. Atribuindo a cada fator os resultados obtidos por suas variáveis originais, é possível tecer algumas considerações acerca de características que desenvolvedores sugerem que os SAD devem apresentar.

1. De acordo com o Fator 1, SAD são sistemas cuja principal característica é propiciar formas de operação dos sistemas através de uma base de dados própria, com possibilidade de definir novos tipos de dados de acordo com a necessidade, e cujas rotinas ou modelos sejam preestabelecidas para solução do problema.
2. O segundo aspecto determinante de um SAD, conforme o Fator 2, é a forma de alimentação do sistema, permitindo acesso a dados internos ou externos à empresa de uma forma amigável.

3. Com menor prioridade, encontra-se a possibilidade de atualização do sistema, com possibilidade autônoma de sua adaptação a novas necessidades (Fator 3).

\section{Usuários}

A aplicação do método de extração das componentes de fatores a partir da matriz de correlações, após rotação Varimax, gerou o carregamento fatorial conforme Figura 3, onde são mostrados os fatores encontrados e seus autovalores.

Os pontos apresentado acima da linha azul na Figura 3 indicam os fatores selecionados (conforme o critério de Kaizer) com autovalor maior que 1. Juntos eles explicam $66,51 \%$ da variância dos dados (Tabela 12). A proporção de variância explicada para o primeiro fator é de 32,94\%; o segundo fator, explica $17,59 \%$; e o terceiro fator, $15,97 \%$. Apesar de possível, um 4o fator não foi considerado, pois o objetivo foi obter o menor número possível de variáveis latentes para a representação.

A Tabela 13 apresenta a carga fatorial com a qual cada variável original se relaciona com cada fator escolhido. Para interpretação desses fatores, foram escolhidas as variáveis com maior peso ou contribuição (ressaltadas).

O Fator 1, que explica 32,9\% da variância dos dados, apresenta maior carga fatorial nas variáveis Grau de amigabilidade

Tabela 10: Fatores, autovalores e \% de variância
total para desenvolvedores.
\begin{tabular}{|c|c|c|}
\hline FATOR & AUTOVALOR & $\%$ DE VARIÂNCIA TOTAL \\
\hline 1 & 2,402 & 34,312 \\
\hline 2 & 1,377 & 19,667 \\
\hline 3 & 1,205 & 17,216 \\
\hline
\end{tabular}

Tabela 11: Cargas fatoriais após rotação Varimax para desenvolvedores.

\begin{tabular}{|l|c|c|c|}
\hline \multicolumn{1}{|c|}{ VARIÁVEL } & FATOR 1 & FATOR 2 & FATOR 3 \\
\hline Origem dos dados & 0,219 & 0,741 & 0,112 \\
\hline Base de dados & 0,890 & $-0,344$ & 0,082 \\
\hline Tipo de dados & 0,871 & 0,195 & $-0,152$ \\
\hline Seqüência de execução & 0,781 & 0,433 & $-0,122$ \\
\hline Grau de adaptação & $-0,118$ & $-0,158$ & 0,792 \\
\hline Grau de amigabilidade & $-0,064$ & 0,737 & $-0,047$ \\
\hline Nível de autonomia & $-0,005$ & 0,192 & 0,756 \\
\hline Autovalor & 2,402 & 1,377 & 1,205 \\
\hline \% de Variância Total & 34,312 & 19,667 & 17,216 \\
\hline
\end{tabular}


da interface do sistema, Base de dados e Nível de autonomia oferecido, que podem definir a manipulação de dados pelo usuário. O Fator 2 explica 17,60\% da variação dos dados e apresenta uma maior contribuição das variáveis Seqüência de execução das rotinas e Origem dos dados, que podem estar relacionadas à operação do sistema. E o Fator 3, com $15,97 \%$ de explicação para variação dos dados, pode ser necessidades, que correspondem a adequação do sistema a novas soluções. avaliado pelas variáveis Tipo de dados e Adaptação a novas

A análise fatorial pôde confirmar o que parece um sentimento lógico, mostrou claramente diferença entre os fatores surgidos a partir dos dados dos desenvolvedores e dos usuários, o que é um indicador da diferença de percepção que têm de SAD. Mais que isso, mostrou que fatores em um grupo não apresentam a mesma prioridade para o outro, podendo indicar um dos causadores de problemas no relacionamento entre eles. O Quadro 4 sintetiza as características priorizadas.

Vê-se que desenvolvedores consideram que SAD são sistemas relacionados primeiramente a operação, visando à manipulação de uma base de dados própria e com flexibilidade na definição dos dados pelo usuário, embora não haja uma preocupação com a utilização de novos modelos. Essa percepção foi destacada nos dados. O segundo aspecto é a alimentação através de dados internos e externos.

Os resultados obtidos apresentam três variáveis latentes, que podem caracterizar SAD pelos usuários. Estas variáveis podem ser denominadas Manipulação, Operação e Adequação. A análise dos fatores, a partir dos resultados obtidos pelas variáveis originais, pode indicar que para os usuários, SAD são sistemas de informação que têm como características prioritárias:

1. Primeiramente, conforme o Fator 1, a possibilidade de manipulação dos dados de uma forma autônoma, agradável e fácil.

2. Propiciar a operação de dados internos e externos à organização, através de rotinas preestabelecidas.

3. Por último, permitir a adequação a outros problemas, através de definição de novos tipos de dados e adaptação a novas necessidades.
A partir de então é que surge o comprometimento com a atualização do sistema, através da sua adaptação a novas necessidades de forma autônoma.

Os usuários, por sua vez, priorizam a manipulação de dados, através de uma interface amigável, que possibilitem autonomia de uso. O segundo aspecto refere-se à forma como o sistema é operado, com rotinas preestabelecidas e

Tabela 12: Fatores, autovalores e \% de variância total para usuários.

\begin{tabular}{|c|c|c|}
\hline FATOR & AUTOVALOR & \% DE VARIÂNCIA TOTAL \\
\hline 1 & 2,306 & 32,941 \\
\hline 2 & 1,232 & 17,596 \\
\hline 3 & 1,118 & 15,970 \\
\hline
\end{tabular}

Figura 3: Autovalores para usuários.

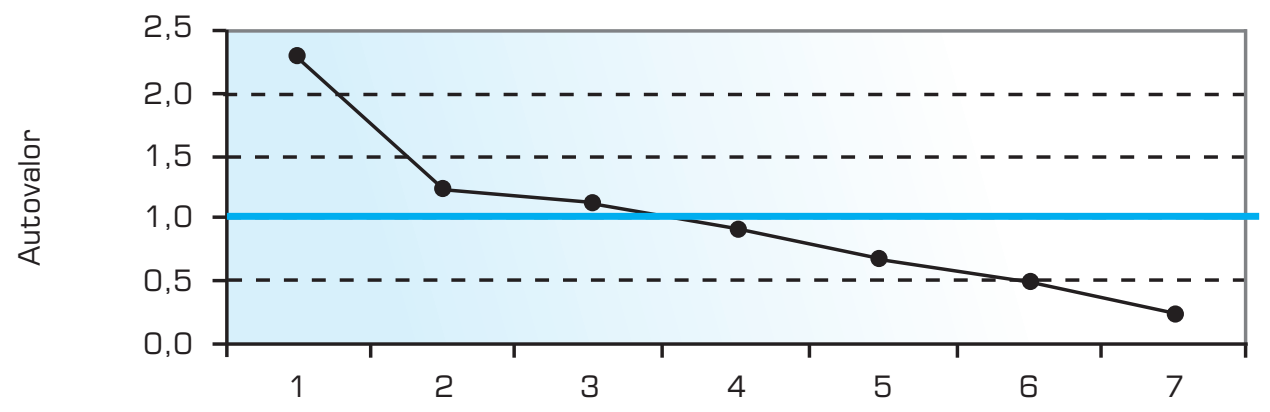

Fator 
Tabela 13: Cargas fatoriais após rotação Varimax para usuários.

\begin{tabular}{|l|c|c|c|}
\hline \multicolumn{1}{|c|}{ VARIÁVEL } & FATOR 1 & FATOR 2 & FATOR 3 \\
\hline Origem dos dados & 0,117 & 0,765 & $-0,295$ \\
\hline Base de dados & 0,764 & $-0,134$ & $-0,153$ \\
\hline Tipo de dados & 0,071 & 0,080 & 0,750 \\
\hline Seqüência de execução & $-0,040$ & 0,843 & 0,282 \\
\hline Grau de adaptação & $-0,467$ & $-0,351$ & 0,574 \\
\hline Grau de amigabilidade & 0,784 & 0,159 & 0,307 \\
\hline Nível de autonomia & 0,707 & 0,220 & $-0,96$ \\
\hline Autovalor & 2,306 & 1,232 & 1,118 \\
\hline \% de Variância Total & 32,941 & 17,596 & 15,970 \\
\hline
\end{tabular}

Quadro 4: Características prioritariamente associadas a SAD.

\begin{tabular}{|l|}
\hline \multicolumn{1}{|c|}{ DESENVOLVEDORES } \\
\hline 1. Operação \\
Base de dados própria \\
Flexibilidade na definição dos dados \\
Execução das rotinas predefinida \\
\hline 2. Alimentação \\
Tratamento de dados internos e externos \\
Interface amigável \\
\hline 3. Atualização \\
Adaptação a novas necessidades \\
Autonomia
\end{tabular}

com acesso a dados internos e externos à empresa. E, por último, a adequação a novas necessidades e que permitam definição de novos tipos de dados.

\section{CONCLUSÃO}

Como apresentada, a percepção que usuários e desenvolvedores (no campo objeto da pesquisa) têm de SAD difere entre si e da literatura. Isso ressalta a importância desta pesquisa, que serve como referência a ações a serem desenvolvidas na formação dos profissionais envolvidos em desenvolvimento e uso de SI, com vistas a proporcionar um bom relacionamento entre eles, bem como sistemas de informação mais eficientes.

Para que os resultados fossem mais abrangentes, a pesquisa foi desenvolvida em destacadas empresas usuárias ou desenvolvedoras de TI. O fato de a pesquisa ter sido enviada e respondida via e-mail demonstra a intenção dos participantes na pesquisa e ressalta que o conteúdo das respostas é espontâneo e corresponde ao que estimam ser SAD.

Deve-se observar, por fim, a limitação do trabalho, que não esgota o assunto, atém-se a alguns aspectos da

\begin{tabular}{|l|}
\hline \multicolumn{1}{|c|}{ USUÁRIOS } \\
\hline 1. Manipulação \\
Interface amigável \\
Base de dados própria \\
Autonomia de uso \\
\hline 2. Operação \\
Seqüência de rotinas predefinida \\
Dados de fontes internas ou externas \\
\hline 3. Adequação \\
Definição flexível de novos tipos de dados \\
Adaptação a novas necessidades \\
\hline
\end{tabular}

questão ampla e complexa, o que indica a necessidade de desenvolvimento de outros estudos nesse campo. Uma das principais dificuldades foi a elaboração do questionário. Conseguir expressar formas de medir se um sistema podia ou não ser considerado SAD sem uma avaliação presencial, foi um grande desafio. Não só pela falta de uma teoria sedimentada de $\mathrm{SAD}$, mas pelo alto nível de abstração de alguns conceitos. Assim, podem ser sugeridos como trabalhos futuros: 1) explorar sob outros pontos de vista a base de dados existentes visando ao estudo de outras questões, de forma a mostrar a riqueza dos dados, tais como: avaliação de desempenho, da eficácia e da usabilidade dos SAD; 2) aprimorar a pesquisa com avaliação dos sistemas in loco para explorar mais a fundo as questões de investigação; 3 ) desenvolver pesquisa para avaliar sistemas e tipificá-los, elaborando um perfil dos SAD existentes.

Em suma, desenvolver SAD requer um maior conhecimento de ferramentas e técnicas que facilitem a criação de sistemas mais flexíveis e a participação efetiva do usuário. $\mathrm{O}$ conhecimento aprofundado do problema e formas para resolvê-lo são mais importantes na construção e uso do SAD. 


\section{Artigo recebido em 29/10/2004 Aprovado para publicação em 06/04/2006}

\section{- Referências Bibliográficas}

ALMEIDA, A.; COSTA A. P.; MIRANDA C. (2002). Informação e gestão. In: ALMEIDA, A.; RAMOS, F. Gestão da informação na competitividade das organizações. 2 . ed, Recife: Editora da UFPE, p. 3-12.

ALTER, S. Decision Support Systems: current practice and continuing challenges. California: Addison-Wesley Publishing Company, 1980

ALTER, S. Transforming DSS jargon into principles for DSS success. In: GRAY, P. Decision support and executive information systems. Englewood Cliffs: Prentice Hall, p. 3-26, 1994

ALVIM, P. C. Bases de um sistema informacional para o apoio à tomada de decisão da inovação em empresas da base tecnológica. Brasília, 219p. Dissertaçnao de Mestrado, Faculdade de Estudos Sociais Aplicados Universidade de Brasília, 2002.

ARTES, R. Aspectos estatísticos da análise fatorial de escalas de avaliação. Revista de Psiquiatria Clínica, 25 (5), Edição Especial: 223-228, 1998. Edição Internet. Disponível em: <http:/www.hcnet.usp. br/ipq/revista/r255/conc255d.htm > Acesso em 21/11/2002.
BARBOSA, G. R. Sistemas de apoio a decisão sob o enfoque de profissionais de TI e decisores. Dissertação de Mestrado, Engenharia de Produção, Universidade Federal de Pernambuco, Recife, 96 p., 2003.

BIDGOLI, H. Decision Support System - Principles and Practice. New York: West Publishing Company, 1989.

CHEN, J. Q. \& LEE, S. M. An exploratory cognitive DSS for strategic decision making. Decision Support Systems n. 36, p. 147-160, 2003.

COURTNEY, J. Decision making and knowledge management in inquiring organizations: toward a new decision-making paradigm for DSS. Decision Support Systems, n. 31, p. 17-38, maio, 2001.

DAVIS, C.; OLSON, M., Management information systems: Conceptual foundations, structure and development. New York: McGraw-Hill, 1974.

FAZLOLLAHI, B.; PARIKH, M. A.; VERMA S. Adaptive decision support systems.
Decision Support Systems, 20, Elsevier Science, 1997.

FINLAY, P. N.; FORGHANI, M. A classification of success factors for decision support systems. Journal of Strategic Information Systems, n. 7, p. 53-70, 1998

FUGLSETH, M.; GRONHAUG, K. Can computerised market models improve strategic decision-making ? An exploratory study. Journal of Socio-Economics, n. 32, p. 503-520, 2003.

GARNTO, C.; WATSON, H. Um levantamento das exigências de bancos de dados para DSS institucionais e Ad hoc. In: SPRAGUE, Jr. ; WATSON, H. Sistem de apoio à decisão: Colocando a teoria em prática. Rio de Janeiro: Campus, $\mathrm{p}$. 156-172, 1991.

HAIR, J. F. et al. Multivariate data analysis. 4. ed., New Jersey: Prentice-Hall, Inc, 1998

LAUDON, K.; LAUDON, J. Sistemas de informação. 4. ed. Rio de Janeiro: Livros Técnicos e Científicos Editora S. 1999.
MATTAR, F. Pesquisa de marketing. São Paulo: Atlas, 1996

POZZEBON, M.; FREITAS, H. Características desejáveis em um EIS - Enterprise Information System - Rumo à proatividade. Revista Eletrônica de Administração, n. 3, junho, 1997.

SANTHANAM, R.; GUIMARAES, T.; GEORGE J. An empirical investigation of ODSS impact on individuals and organizations. Decision Support Systems, n. 30, p. 51-72, 2000 .

SELLTIZ, C.; JAHODA, M.; DEUTCH, M. COOK, S. Método de pesquisa nas relações sociais. São Paulo: Editora da Universidade de São Paulo, 1967.

SPRAGUE, Jr.; WATSON, H. Decision support systems: putting theory into practice. USA: Prentice-Hall, 1989.

STAIR, R. Princípios de sistemas de informação - Uma abordagem gerencial. 2. ed., Rio de Janeiro: LTC, 1998.

\section{- Sobre os autores}

Gilka Rocha Barbosa

UFPE - Universidade Federal de Pernambuco

E-mail: gilka@unicap.br

\section{Adiel Teixeira de Almeida}

UFPE - Universidade Federal de Pernambuco

Prof. Adjunto - Departamento de Engenharia de Produção

E-mail: aalmeida@ufpe.br

\section{Ana Paula Cabral Seixas Costa}

UFPE - Universidade Federal de Pernambuco

Prof. Adjunto - Departamento de Engenharia de Produção

E-mail: apcabral@ufpe.br 